\title{
Evidências de validade do Experience in Close Relationships (ECR) Inventory para o Brasil
}

\author{
Victor Kenji Medeiros Shiramizu \\ Universidade Federal do Rio Grande do Norte \\ Jean Carlos Natividade \\ Universidade Federal do Rio Grande do Sul \\ Fívia de Araújo Lopes \\ Universidade Federal do Rio Grande do Norte
}

\begin{abstract}
Resumo
Instrumentos que aferem apego adulto fundamentam-se na noção de que os padrões de vinculação estabelecidos na primeira infância transpõem-se para relacionamentos amorosos na vida adulta. O objetivo desta pesquisa foi buscar evidências de validade de um instrumento para aferir apego adulto, traduzido e adaptado para o Brasil: Experience in Close Relationships Inventory (ECR). Para tanto, realizaram-se dois estudos consecutivos. No primeiro, traduziu-se o ECR e aplicou-se em 230 estudantes universitários. A partir dos resultados, modificaram-se itens e aplicou-se a escala em uma amostra de 618 pessoas das cinco regiões do Brasil. Os resultados indicaram uma estrutura bidimensional, ansiedade e evitação relacionada ao apego, para o ECR-Brasil, bem como, adequados índices de precisão. Encontraram-se correlações consonantes com a teoria do construto para as duas dimensões avaliadas, níveis de amor e sociossexualidade. O instrumento também diferenciou pessoas em relacionamento compromissado e não compromissado. Considera-se que ECR-Brasil mostrou satisfatórias evidências de validade e precisão.
\end{abstract}

Palavras-chave: Teoria do Apego; amor; testes psicológicos.

\begin{abstract}
Validate evidences of Experience in Close Relationships (ECR) Inventory to Brazil. Instruments that assess adult attachment are based on the notion that patterns of bonding established in early childhood transpose to romantic relationships in adult life. The aim of this research was to seek validity evidences of an instrument to assess adult attachment, translated and adapted to Brazil: Experience in Close Relationships Inventory (ECR). Two consecutive studies were performed. In the first the ECR was translated and responded by 230 college students. From the results, items were changed and the scale was responded by 618 people from five regions of Brazil. The results indicated a two-dimensional structure to ECR-Brazil, attachment-related anxiety and avoidance, as well as appropriate indices of reliability. Correlations in line with the theory have been found among the two-dimensional construct, levels of love and sociosexuality. The instrument also differentiated people in committed and non-committed relationship. We consider that ECR-Brazil showed satisfactory evidence of validity and reliability.
\end{abstract}

Keywords: Attachment Theory; love; psychological testing.

\section{Resumen}

Evidencias de validez del Experience in Close Relationships (ECR) Inventory a Brasil. Instrumentos para evaluar apego adulto se basan en la idea de que los patrones de vinculación establecidos en la primera infancia transponen a las relaciones amorosas en la vida adulta. El objetivo de esta investigación fue buscar evidencias de validez de un instrumento para evaluar el apego adulto, traducido y adaptado a Brasil: Experience in Close Relationships Inventory (ECR). Se realizaron dos estudios consecutivos. En el primero el ECR se tradujo y se respondió con 230 estudiantes universitarios. A partir de los resultados, ítems fueron cambiados y 618 personas de cinco regiones de Brasil respondieron la escala. Los resultados indicaron una estructura de dos dimensiones para ECR-Brasil, así como índices apropiados de confiabilidad. Se han encontrado correlaciones en consonancia con la teoría entre la construcción de dos dimensiones, la ansiedad y la evitación, y los niveles de amor y sociosexualidad. El instrumento también diferenció las personas en una relación comprometida y no comprometida. Consideramos que la ECR-Brasil mostró evidencia satisfactoria de validez y confiabilidad.

Palabras clave: Teoría del apego; amor; tests psicológicos. 


\section{As bases da teoria do apego}

Até a década de 1950 a perspectiva vigente sobre o estabelecimento de vínculos afetivos entre a criança e seus cuidadores discorria sobre a primazia do fornecimento de alimentação como o meio de ligação entre filhos e pais. Essa visão, mais bem representada pela teoria do amor interesseiro das relações objetais, dominava os escritos psicanalíticos à época (Bowlby, 1969/2002). Com a publicação dos estudos de Harry Harlow com macacos rhesus, a importância do contato e conforto entre criança e cuidador passou a ser evidenciada. Harlow (1958) demonstrou como a qualidade da relação entre o cuidador e o ser cuidado seria de fundamental importância para o desenvolvimento psicossocial adequado do indivíduo. Concomitantemente, John Bowlby estabeleceu uma relação entre crianças que possuíam histórias de perda ou separação materna e foram criadas em orfanatos e uma maior susceptibilidade a desenvolver depressão e problemas comportamentais e emocionais (Chisholm, 1996).

Em sua trilogia Apego e Perda, Bowlby combinou ideias da psicanálise, da psicologia do desenvolvimento, da teoria dos sistemas de controle e da etologia, propondo que humanos são capazes de desenvolver vínculos emocionais durante o primeiro ano de vida através do sistema comportamental de apego (Bretherton, 1992). Esse sistema teria sido moldado pela seleção natural, durante o Ambiente de Adaptação Evolutiva (AAE) de nossa espécie, para promover segurança e aumentar as chances de sobrevivência do indivíduo (Fraley \& Shaver, 2000).

Bowlby afirmava que, através das diversas interações com o cuidador principal, a criança seria capaz de elaborar modelos funcionais internos de si e do outro, os quais perdurariam ao longo da vida. O primeiro modelo funcional referia-se a quanto o indivíduo acreditava ser aceitável aos olhos da figura de apego. Já o modelo funcional do outro dizia respeito a quanto o indivíduo acreditava que a figura de apego estaria disponível e receptiva quando fosse necessário (Bowlby, 1973/2004). Assim, a figura de apego serviria tanto como base segura (secure base), a partir da qual a criança se sentiria segura para explorar o ambiente; bem como refúgio seguro (safe haven), nos momentos em que ela se sentisse angustiada ou assustada durante a exploração (Bowlby, 1969/2002; Chisholm, 1996).

A participação da pesquisadora Mary Ainsworth na equipe de Bowlby foi essencial para o estudo empírico da variação existente na qualidade da relação mãe-bebê. Ainsworth, Blehar, Waters e Wall (1978) desenvolveram um procedimento padronizado denominado Situação Estranha. Esse procedimento, estruturado em ambiente de laboratório, tinha como objetivo ativar, de forma gradativa, o sistema comportamental de apego da criança baseado em duas separações da mãe, juntamente com a presença de um estranho. A partir de resultados de experimentos em que esse procedimento foi utilizado, Ainsworth et al. (1978) perceberam que era possível categorizar as crianças em três grupos de acordo com o estilo de apego: aquelas com apego seguro, as com apego inseguro evitativo e as com apego inseguro ansioso/ambivalente.

Alguns anos mais tarde, pesquisadores da Universidade de Berkeley se depararam com a dificuldade de classificar o padrão de apego observado em algumas crianças. Através de análises de vídeos de aproximadamente 200 crianças durante a Situação Estranha, Main e Solomon (1986) observaram comportamentos extremamente estereotipados e grande medo do cuidador principal durante o procedimento. Esses sinais não eram observados quando o cuidador estava ausente. Além disso, outra principal característica encontrada nessas crianças foi a ausência de estratégias coerentes para enfrentar eventos estressores, tais como a Situação Estranha. Assim, as pesquisadoras concluíram que era possível categorizá-las dentro de um novo padrão de apego: o inseguro desorganizado/desorientado (Main \& Solomon, 1986).

\section{Apego adulto: Uma continuidade do vínculo criança- cuidador}

Em 1987, Cindy Hazan e Phillip Shaver, exploraram a possibilidade do amor romântico em adultos estar fundamentado em um processo de apego. Segundo tais autores, os modelos funcionais internos propostos por Bowlby (estabelecidos com o cuidador principal durante as diversas interações na infância) continuariam a guiar e moldar o comportamento do indivíduo na idade adulta. Dessa forma, tais modelos poderiam ser extrapolados para outras relações interpessoais, como por exemplo, as relações românticas. Isso porque tanto na interação cuidador principal-criança quanto na existente entre parceiros românticos há formação de vínculos afetivos, noção de segurança e conforto quando a figura de apego está disponível e angústia quando há separação desta (Fraley \& Shaver, 2000; Hazan \& Shaver, 1987).

Para mensurar apego adulto, no contexto das relações românticas, Hazan e Shaver traduziram componentes chave da teoria do apego, adequando-os para adultos, focando nos três estilos de apego propostos por Ainsworth et al. (1978) e utilizaram um questionário no qual havia três frases para que o respondente escolhesse a que ele mais concordava. Cada uma dessas frases correspondia a um estilo de apego: seguro, inseguro evitativo e inseguro ansioso/ambivalente. Um dos resultados encontrados foi a distribuição de participantes em cada categoria de apego muito semelhante à de Ainsworth et al. (1978) para apego infantil, reforçando a continuidade dos modelos mentais estabelecidos na infância até a idade adulta.

Após a publicação do trabalho de Hazan e Shaver (1987), diversos estudos surgiram no intuito de observar o que era próprio de cada estilo de apego dentro de uma relação amorosa/ romântica (ver Belsky, 1997, para uma revisão). Resumidamente, os achados têm sido semelhantes no sentido de indicar uma associação entre comprometimento, confiança, níveis de amor e satisfação com relacionamento entre aqueles com apego seguro; e padrões opostos entre aqueles com estilo inseguro, sobretudo entre os evitativos (Collins \& Read, 1990; Madey \& Rodgers, 2009; Simpson, 1990). Além disso, as pessoas com apego seguro, comparadas às com apego inseguro, têm relacionamentos mais duradouros e compromissados e são mais restritas sexualmente (Hazan \& Shaver, 1987; Schmitt, 2005).

Todavia, limitações e críticas ao modelo de avaliação de Hazan e Shaver surgiram, sendo as mesmas reconhecidas pelos próprios autores. Primeiro devido ao modelo focar apenas uma 
via da relação, ou seja, avaliava-se apenas o modo como o indivíduo se via (Hazan \& Shaver, 1987). Segundo, pelo fato da medida utilizada ser de escolha forçada, o que tornava a classificação categórica, ou seja, não era possível estabelecer graduações entre os estilos de apego (Feeney, 2008). Ainda, esse modelo de autoavaliação poderia não ser tão preciso para obter informações necessárias dos participantes, justamente pela ausência de multi-itens, os quais poderiam facilitar a avaliação de diferenças individuais (essência da teoria do Apego) (Fraley $\&$ Shaver, 2000).

Outras medidas de apego adulto foram elaboradas, por exemplo, Bartholomew e Horowitz (1991) propuseram avaliar os estilos de apego em quatro categorias ao invés de três. Esses autores formularam quatro frases que levavam em consideração a imagem abstrata que o indivíduo possuía de si - modelo interno de si (positivo ou negativo) e a imagem abstrata que o indivíduo possuía de outra pessoa - modelo interno do outro (positivo ou negativo). Sob essa perspectiva o estilo inseguro evitativo foi desmembrado em: evitativo medroso e evitativo rejeitador; resultando em quatro estilos de apego (Bartholomew \& Horowitz, 1991). Esse modelo aproxima-se da proposta de Main e Solomon (1986) quanto à classificação dos estilos de apego.

Posteriormente, Griffin e Bartholomew (1994) criaram o Questionário dos Estilos das Relações (Relationships Questionnaire Styles). Essa escala, de natureza multi-item, composta por 30 itens, permite avaliar o indivíduo tanto categoricamente quanto dimensionalmente. Ainda, tal escala demonstrou uma maior confiabilidade do que o instrumento proposto em 1991 por Bartholomew e Horowitz, pois a classificação seria baseada não apenas em uma única frase, mas a partir de um conjunto de afirmações (Crowell, Fraley, \& Shaver, 2008). A proposta de Griffin e Bartholomew (1994) chamou a atenção de pesquisadores, que passaram a ver que o apego seria mais bem compreendido por meio de dimensões. Com essa noção de um construto dimensional Brennan,Clark e Shaver (1998) desenvolveram uma escala que tem sido bastante utilizada e adaptada a diversas culturas, sobretudo por suas propriedades psicométricas e adequação conceitual à teoria: Experience in Close Relationships Inventory.

\section{Experience in Close Relationships Inventory (ECR)}

O ECR é um instrumento que se propõe a medir apego adulto a partir da interação estabelecida em relacionamentos amorosos. Ele é derivado de um levantamento de itens de instrumentos de apego adulto disponíveis na literatura na década de 1990 que resultou num conjunto inicial de 323 itens (Brennan et al., 1998). Os autores aplicaram esse conjunto de itens a mais de 1200 participantes e, a partir de uma análise fatorial, concluíram que a solução mais adequada seria extrair dois fatores, denominados de Ansiedade relacionada ao apego e Evitação relacionada ao apego. Posteriormente, os pesquisadores selecionaram um pequeno conjunto de itens, os quais tiveram as cargas fatoriais mais altas em cada dimensão para compor uma versão final do instrumento. O ECR é composto por 36 itens que estão divididos igualmente entre as duas dimensões: 18 para Ansiedade e 18 para Evitação. A primeira dimensão diz respeito ao quanto o indivíduo preocupase com a responsividade do parceiro e com o relacionamento, bem como o quanto ele necessita de proximidade física e emocional. Já a segunda dimensão diz respeito ao desconforto com a proximidade emocional, com a dependência de parceiros românticos, uma preferência por distanciamento emocional, baixa emocionalidade e extrema autoconfiança. A versão original do instrumento apresentou coeficiente alfa de Cronbach de 0,91 para a dimensão Ansiedade relacionada ao Apego e 0,94 para a dimensão Evitação relacionada ao Apego (Brennan et al., 1998).

Esse instrumento tem sido traduzido e adaptado para diversas populações, como por exemplo: italiana (Piccardi, Bitetti, \& Puddu, 2000), chinesa (Mallinckrodt \& Wang, 2004), espanhola (Alonso-Arbiol, Balluerka, \& Shaver, 2007) e portuguesa (Paiva \& Figueiredo, 2010). Apesar disso, até o momento da realização deste manuscrito, não foram encontrados estudos demonstrando evidências de validade do ECR para a população brasileira. Ainda que não haja estudos que demonstrem adequações psicométricas do ECR para o Brasil, o estudo de apego adulto tem despertado o interesse de pesquisadores no país, uma vez que a Teoria do Apego é aplicada para uma melhor compreensão da dinâmica dentro das relações interpessoais (por exemplo, Basso \& Marin, 2010; Fonseca, Silva, \& Otta, 2010).

\section{Caracterização da pesquisa}

Esta pesquisa teve por objetivo traduzir, adaptar e apresentar evidências de validade e precisão de uma escala delineada para aferir apego em adultos, no contexto das relações românticas. Nomeadamente, buscou-se desenvolver uma versão brasileira do Experience in Close Relationships (ECR) Inventory (Brennan et al., 1998).

Trata-se de uma pesquisa conduzida em dois momentos. No primeiro estudo realizou-se a tradução dos itens do ECR e aplicou-se em uma amostra de universitários. No Estudo 2 ajustaram-se os itens que se mostraram problemáticos no primeiro estudo e aplicou-se o instrumento em pessoas de todas as regiões do Brasil. Além das propriedades psicométricas do instrumento, buscaram-se relações de apego com amor, no modelo triangular de Sternberg (1997), e sociossexualidade (Penke \& Asendorpf, 2008; Simpson \& Gangestad, 1991).

\section{Método}

Estudo 1

\section{Tradução e adaptação dos itens do ECR-Brasil}

Encaminhou-se a escala original em inglês, independentemente, para três especialistas bilíngues (portuguêsinglês), todos brasileiros natos e com conhecimento avançado na língua inglesa. Cada um dos especialistas traduziu para o português todos os itens do ECR. Os pesquisadores compilaram as versões dos especialistas em uma única versão levando-se em conta o consenso entre os especialistas e a adequação dos termos ao cotidiano e realidade local. Em uma segunda etapa, apresentou-se a versão consensual a 15 estudantes de graduação (cursos de Psicologia e Ciências Biológicas) e solicitou-se que avaliassem a compreensão e, quando julgassem necessário, sugerissem reformulações. Após realizarem-se ajustes de acordo 
com as sugestões dos estudantes, concluiu-se uma versão do ECR-Brasil para ser posta ao teste empírico.

\section{Participantes}

Participaram 230 estudantes provenientes de três universidades da grande Natal $(\mathrm{RN})$, com idade entre $18 \mathrm{e}$ 46 anos $(M=22,9$ anos; $D P=4,62)$, sendo $68,7 \%$ mulheres. Ainda, 59,6\% $(N=137)$ dos participantes estavam em um relacionamento compromissado. Não houve restrição quanto à orientação sexual dos participantes.

\section{Instrumentos}

Utilizou-se um questionário com perguntas sociodemográficas, tais como sexo e idade, e a versão traduzida do ECR. A apresentação da escala foi idêntica a da versão original, ou seja, o que era o item 1 na escala original mantevese item 1 na versão traduzida e assim por diante. $O$ formato para as respostas ao ECR também foi idêntico ao utilizado na versão original do instrumento, qual seja: os participantes deveriam responder em uma escala de sete pontos o quanto concordavam com os itens, em que o ponto 1 correspondia a 'discordo fortemente' e o 7, a 'concordo fortemente'. $\mathrm{Na}$ consigna instruía-se os respondentes a pensarem em como eles geralmente vivenciavam seus relacionamentos, não apenas sobre o que estava acontecendo em um possível relacionamento atual.

\section{Procedimentos}

As coletas de dados foram realizadas de modo coletivo, durante o período de aulas nas universidades. Os participantes foram instruídos pelos pesquisadores a respeito da pesquisa e forma de responder ao questionário. Todos os procedimentos éticos foram seguidos em conformidade com a resolução 196/96 do Conselho Nacional de Saúde.

\section{Resultados}

Inicialmente, verificou-se a presença de 10 casos omissos nas respostas aos itens referentes à ECR, os quais foram substituídos pelas médias da série. Em seguida, executou-se uma análise de componentes principais com método de rotação oblimin. Utilizou-se esse método por ele ter sido utilizado no estudo inicial de elaboração do instrumento (Brennan et al., 1998) e porque essa rotação não impede a emergência de dimensões ortogonais (Pasquali, 2005). Verificou-se adequação dos dados à fatorização, $K M O=0,84$, bem como da matriz de correlações dos itens, teste de esfericidade de Bartlett: $\chi^{2}(630, N=230)=3036 ; p<$ 0,001 . Foram encontradas oito dimensões com eigenvalue $>1$, com os seguintes eigenvalues: 6,$49 ; 5,82 ; 1,94 ; 1,67 ; 1,48 ; 1,44$; 1,09 e 1,02. A discrepância entre as duas primeiras dimensões e as demais, juntamente com o embasamento teórico sobre um construto de dois fatores do instrumento original (Brennan et al., 1998), apontam para a adequação da extração de duas dimensões. Forçando-se duas dimensões, a variância total explicada pelos itens foi de $34,2 \%$. A correlação entre as dimensões foi de 0,04 . As cargas componenciais dos itens em cada dimensão e demais propriedades psicométricas são apresentadas na Tabela 1 .

A maior parte dos itens mostrou-se em acordo com a versão original da escala, contudo, foram encontrados problemas em pelo menos dois itens: o item 13, que originalmente refere-se ao fator Evitação e apresentou carga superior na dimensão Ansiedade; e o item 29, que apresentou cargas semelhantes e baixas em ambas as dimensões. Apesar dos problemas apresentados, os coeficientes alfa (Cronbach, 1951), calculados com os itens de acordo com a versão original, foram de 0,87 em ambas as dimensões.

\section{Método}

Estudo 2

\section{Ajustes à versão preliminar}

A partir dos resultados do Estudo 1, voltou-se à escala original e comparou-se o conteúdo de cada item traduzido. Realizaram-se ajustes a 22 itens da versão anterior com vistas a aperfeiçoar o instrumento. As modificações incluíram substituição de termos, reescrita de itens e acréscimos de expressões. Por exemplo, o item 13 mudou de "Eu fico ansioso(a) quando minhas(meus) parceiras(os) chegam muito próximo de mim" para "Eu fico apreensivo quando uma(um) parceira(o) fica muito próxima(o) afetivamente de mim”. Ao item 29 também se acrescentou o termo "afetivamente", considerando-se o caráter econômico vinculado à palavra "depender", no Brasil (versão final apresentada no Apêndice 1).

\section{Participantes}

Participaram 618 pessoas, média de idade de 25,9 anos (DP $=7,09), 59,2 \%$ eram mulheres. A escolaridade variou de ensino médio a ensino de pós-graduação: $1,8 \%$ tinham até o ensino médio completo, $44,7 \%$ estavam cursando o ensino superior e $53,5 \%$ tinha o ensino superior completo. Houve participantes de todas as regiões do Brasil, sendo a região Nordeste a mais representativa com $64,8 \%$ dos participantes, a região Sul teve $21,3 \%$ dos participantes, a Sudeste $7,7 \%$, a Centro-Oeste $3,1 \%$ e a Norte $2,6 \%$.

O tempo do relacionamento amoroso mais longo que os participantes já estabeleceram variou de um a 420 meses $(M$ $=51,1 ; D P=55,2)$. No que diz respeito à situação conjugal atual, 73,9\% $(N=457)$ dos participantes afirmaram estar em um relacionamento amoroso. Dentre esses em relacionamento $89,7 \%(N=410)$ estavam em um relacionamento compromissado (casamento, união estável, noivado, namoro) e os 10,3\% $(N=47)$ demais em um relacionamento não compromissado ("ficando", "amizade colorida", "rolo", apenas sexo). O tempo de duração do relacionamento atual variou de um dia a 408 meses $(M=$ $46,2 ; D P=59,2)$

\section{Instrumentos}

Utilizou-se um questionário on-line autoaplicável, semelhante a um questionário lápis-papel, porém disponibilizado em um endereço na internet. O questionário continha perguntas sociodemográficas, tais como: sexo, idade, escolaridade e Estado brasileiro em que residia. Também havia questões sobre relacionamentos amorosos, a saber: tempo do relacionamento amoroso mais longo que já teve na vida; se estava em um 
Tabela 1

Cargas Componenciais dos Itens da ECR-Brasil a partir de uma Análise de Componentes Principais com Rotação Oblimim e demais Propriedades Psicométricas

\begin{tabular}{|c|c|c|c|c|c|c|}
\hline & \multicolumn{3}{|c|}{ Estudo 1} & \multicolumn{3}{|c|}{ Estudo 2} \\
\hline & Ansiedade & Evitação & $h^{2}$ & Ansiedade & Evitação & $h^{2}$ \\
\hline Item 6 (Ansiedade) & $\mathbf{0 , 5 3}$ & 0,17 & 0,30 & 0,73 & 0,03 & 0,54 \\
\hline Item 8 (Ansiedade) & 0,33 & 0,07 & 0,11 & 0,71 & $-0,12$ & 0,53 \\
\hline Item 24 (Ansiedade) & $\mathbf{0 , 5 7}$ & 0,16 & 0,34 & 0,70 & 0,06 & 0,50 \\
\hline Item 4 (Ansiedade) & 0,54 & $-0,20$ & 0,34 & 0,70 & $-0,10$ & 0,50 \\
\hline Item 18 (Ansiedade) & 0,72 & $-0,02$ & 0,51 & 0,69 & $-0,08$ & 0,49 \\
\hline Item 30 (Ansiedade) & 0,61 & $-0,17$ & 0,42 & 0,68 & $-0,16$ & 0,50 \\
\hline Item 36 (Ansiedade) & 0,62 & $-0,13$ & 0,40 & 0,67 & $-0,07$ & 0,46 \\
\hline Item 2 (Ansiedade) & 0,64 & 0,09 & 0,42 & 0,64 & $-0,04$ & 0,41 \\
\hline Item 10 (Ansiedade) & 0,47 & $-0,10$ & 0,23 & 0,62 & $-0,17$ & 0,42 \\
\hline Item 22 (Ansiedade) & $-0,49$ & 0,09 & 0,25 & $-0,60$ & 0,05 & 0,37 \\
\hline Item 14 (Ansiedade) & 0,64 & $-0,09$ & 0,42 & 0,59 & 0,05 & 0,35 \\
\hline Item 20 (Ansiedade) & 0,64 & 0,00 & 0,41 & 0,59 & 0,05 & 0,35 \\
\hline Item 32 (Ansiedade) & $\mathbf{0 , 5 8}$ & $-0,07$ & 0,35 & 0,59 & $-0,13$ & 0,37 \\
\hline Item 16 (Ansiedade) & 0,51 & 0,11 & 0,26 & 0,57 & $\mathbf{0 , 3 2}$ & 0,41 \\
\hline Item 34 (Ansiedade) & 0,48 & $-0,10$ & 0,25 & 0,56 & $-0,06$ & 0,32 \\
\hline Item 26 (Ansiedade) & 0,58 & 0,22 & 0,37 & 0,54 & 0,37 & 0,41 \\
\hline Item 12 (Ansiedade) & 0,60 & $-0,05$ & 0,37 & 0,52 & 0,27 & 0,33 \\
\hline Item 28 (Ansiedade) & 0,46 & $-0,01$ & 0,21 & 0,43 & 0,06 & 0,19 \\
\hline Item 23 (Evitação) & 0,01 & 0,60 & 0,37 & 0,13 & 0,75 & 0,57 \\
\hline Item 13 (Evitação) & $\mathbf{0 , 5 1}$ & 0,35 & 0,37 & 0,10 & 0,70 & 0,50 \\
\hline Item 1 (Evitação) & 0,07 & 0,58 & 0,34 & 0,13 & 0,70 & 0,49 \\
\hline Item 5 (Evitação) & 0,17 & 0,61 & 0,40 & 0,10 & 0,69 & 0,48 \\
\hline Item 17 (Evitação) & 0,05 & 0,63 & 0,40 & 0,11 & 0,65 & 0,43 \\
\hline Item 11 (Evitação) & $\mathbf{0 , 3 3}$ & 0,62 & 0,47 & 0,30 & 0,63 & 0,48 \\
\hline Item 15 (Evitação) & 0,02 & $-0,66$ & 0,44 & 0,07 & $-0,62$ & 0,40 \\
\hline Item 9 (Evitação) & 0,03 & 0,60 & 0,35 & 0,11 & 0,62 & 0,39 \\
\hline Item 7 (Evitação) & $-0,05$ & 0,64 & 0,41 & 0,04 & 0,61 & 0,37 \\
\hline Item 3 (Evitação) & 0,01 & $-0,57$ & 0,32 & 0,16 & $-0,60$ & 0,39 \\
\hline Item 27 (Evitação) & 0,04 & $-0,68$ & 0,46 & 0,10 & $-0,60$ & 0,37 \\
\hline Item 25 (Evitação) & 0,09 & $-0,65$ & 0,43 & 0,12 & $-0,59$ & 0,36 \\
\hline Item 35 (Evitação) & 0,27 & $-0,57$ & 0,41 & 0,29 & $-0,54$ & 0,39 \\
\hline Item 31(Evitação) & 0,01 & $-0,56$ & 0,31 & 0,11 & $-0,54$ & 0,31 \\
\hline Item 33 (Evitação) & 0,16 & $-0,47$ & 0,25 & 0,20 & $-0,53$ & 0,32 \\
\hline Item 19 (Evitação) & $-0,19$ & $-0,47$ & 0,24 & $-0,13$ & $-0,53$ & 0,29 \\
\hline Item 21 (Evitação) & $-0,23$ & $\mathbf{0 , 3 9}$ & 0,22 & $-0,09$ & 0,35 & 0,13 \\
\hline Item 29 (Evitação) & 0,23 & $-0,30$ & 0,15 & 0,16 & $-0,31$ & 0,12 \\
\hline Eigenvalue inicial & 6,49 & 5,82 & & 7,45 & 6,80 & \\
\hline \% Variância Explicada & 18,00 & 16,20 & $34,2 \%$ & 20,70 & 18,90 & $39,6 \%$ \\
\hline Média & 4,15 & 3,00 & & 4,13 & 2,72 & \\
\hline Desvio-Padrão & 1,06 & 0,97 & & 1,15 & 0,96 & \\
\hline Coeficiente Alfa & 0,87 & 0,87 & & 0,91 & 0,89 & \\
\hline
\end{tabular}

Nota. Itens que compuseram a dimensão Ansiedade: 6, 8, 24, 4, 18, 30, 36, 2, 10, 22, 14, 20, 32, 16, 34, $26,12,28$. Itens que compuseram a dimensão Evitação: 23, 13, 1, 5, 17, 11, 15, 9, 7, 3, 27, 25, 35, 31, 33, 19, 21, 29. Cargas componenciais maiores que 0,30 estão em negrito. Os itens estão apresentados em ordem decrescente a partir das cargas componenciais do Estudo 2. A numeração dos itens segue o mesmo padrão da ECR original de Brennan et al. (1998). Os nomes entre parênteses correspondem às dimensões em que os itens carregaram no instrumento original. 
relacionamento amoroso no momento da pesquisa; como classificava seu relacionamento dentre as opções casado, união estável, noivado, namoro, rolo, ficando, amizade colorida, apenas sexo; o tempo de duração do relacionamento atual. O questionário também incluía o ECR-Brasil com os ajustes realizados a partir do Estudo 1 e as seguintes escalas:

Escala Triangular do Amor de Sternberg (versão brasileira de Cassepp-Borges \& Teodoro, 2007). Essa escala mede níveis de amor a partir de três dimensões: intimidade, comprometimento e paixão. Ela foi traduzida e adaptada para o Brasil a partir da versão original de Sternberg (1997), apresentando satisfatórias evidências de validade e precisão, os coeficientes alfas variaram de 0,90 a 0,91. A escala reduzida é composta por 18 itens, seis para cada dimensão do amor, em que os participantes devem responder em uma escala de nove pontos o quanto consideram que cada item os descreve adequadamente.

Inventário de Orientação Sociossexual-Revisado - SOI-R Brasil (J. C. Natividade, comunicação pessoal, 2012). Esse instrumento afere níveis de irrestrição sexual a partir de três dimensões: comportamento, atitude e desejo. Trata-se de uma versão traduzida e adaptada para o Brasil do Revised Sociosexual Orientation Inventory (Penke \& Asendorpf, 2008). $\mathrm{O}$ instrumento conta com nove itens com respostas em formato de escala de cinco pontos, tal que quanto maiores os escores, maior irrestrição sexual. A tradução e adaptação do instrumento à realidade brasileira contaram com participantes das cinco regiões do país $(N=1887)$, apresentou satisfatórias evidências de validade e coeficientes alfa variando de 0,85 a 0,89 .

\section{Procedimentos}

Os participantes foram recrutados via e-mails-convites e anúncios-convites disponibilizados em redes sociais. Os convites continham o link para o questionário on-line que ficou disponível para respostas por dois meses. O questionário foi configurado para não permitir casos omissos às respostas do ECR. Incluíram-se nas análises todos os participantes que completaram o questionário.

\section{Resultados}

Estudo 2

\section{Estrutura e fidedignidade}

Como no Estudo 1, inicialmente, realizou-se uma análise de componentes principais com rotação oblimin. Constatouse adequação dos dados à análise, $K M O=0,92$ e teste de esfericidade de Bartlett: $\chi^{2}(630, N=618)=9272 ; p<0,001$. Foram encontradas seis dimensões com eigenvalue $>1$, seguem os respectivos eigenvalues: 7,$45 ; 6,80 ; 1,76 ; 1,58 ; 1,34 ; 1,18$. Forçou-se a extração de duas dimensões, em acordo com o instrumento original (Brennan et al., 1998), as quais explicaram $39,6 \%$ da variância dos dados. A primeira dimensão agrupou os 18 itens referentes à Ansiedade e a segunda os 18 itens referentes à Evitação, ambas com os itens carregando de acordo com a versão original do ECR, como pode ser visto na Tabela 1. A correlação entre as dimensões foi de 0,03 . Os cálculos de consistência interna do instrumento (Cronbach, 1951) foram superiores a 0,80 em todas as dimensões e sugerem adequada precisão do instrumento (Nunnally, 1978).

Adicionalmente, a fim de verificar o ajuste dos dados à estrutura bifatorial do instrumento, realizou-se uma análise fatorial confirmatória. Para a especificação do modelo a ser testado, utilizou-se o procedimento de desagregação parcial (Bagozzi \& Heatherton, 1994) para formar seis variáveis explicativas de cada um dos traços latentes do modelo. Desse modo, o modelo configurou-se com as duas variáveis latentes correlacionadas, Evitação e Ansiedade, e cada uma delas explicada por seis observáveis, tais que cada uma das observáveis foi composta pelo agrupamento de três itens aleatoriamente escolhidos de dentro de suas respectivas dimensões. Esse procedimento foi idêntico ao de outros estudos que testaram a estrutura fatorial do ECR revisado (Brenning, Soenens, Braet, \& Bosmans, 2011; Sibley, Fisher, \& Liu, 2005).

Para estimar os parâmetros partiu-se da matriz de covariância dos dados e optou-se pelo algoritmo Maximum Likelihood. Utilizou-se como critério de adequação dos dados ao modelo os seguintes índices, os quais têm sido apontados como aceitáveis pela literatura especializada (Byrne, 2009; McDonald \& Ho, 2002): razão entre qui-quadrado e graus de liberdade < 5; tendo-se obtido $\chi^{2}(53, N=618)=252,2 ; p<0,001$ e $\chi^{2} / D F=$ 4,76; Goodness-of-Fit Index (GFI) >0,90, tendo-se obtido GFI $=0,94 ;$ Adjusted Goodness-of-Fit Index (AGFI) > 0,90, tendo-se obtido AGFI = 0,91; Comparative Fit Index (CFI) $>0,90$, tendose obtido CFI $=0,95$; Normed Fit Index (NFI) $>0,90$, tendo-se obtido NFI = 0,94; Root Mean Square Error of Aproximation (RMSEA) $<0,08$, tendo-se obtido RMSEA $=0,078$ (Intervalo de Confiança de $90 \%=0,069-0,088)$. Esses índices, tomados em conjunto, sugerem adequação da estrutura do instrumento (Byrne, 2009; McDonald \& Ho, 2002).

\section{Correlações com amor e sociossexualidade}

Testaram-se as correlações entre as dimensões Ansiedade e Evitação de apego e as demais variáveis do estudo, como podem ser vistas na Tabela 2. Observaram-se correlações significativas entre cada uma das variáveis estudadas com pelo menos uma dimensão de apego. Entre as correlações mais fortes, destacam-se as de Evitação com os três fatores do amor (intimidade, paixão e comprometimento), sendo essas negativas. Todas as correlações, ainda que algumas tenham sido fracas, foram no sentido esperado pela teoria que embasa o instrumento.

\section{Poder discriminativo}

Considerando que a variável idade mostrou-se significativamente correlacionada com a dimensão Ansiedade, controlou-se o efeito daquela variável em todas as análises subsequentes. Inicialmente testaram-se diferenças sexuais em apego adulto por meio de uma MANOVA. Constatou-se diferença significativa entre sexo, $\Lambda$ de Wilks $=0,95 ; F(2,609)$ $=16,6 ; p<0,001 ; \eta^{2}=0,05$. Os testes univariados revelaram que para a dimensão Ansiedade as mulheres $(M=4,29 ; D P$ $=1,09)$ apresentaram pontuação significativamente maior do que os homens $(M=3,91 ; D P=1,20), F(1,610)=16,9 ; p<$ 0,$001 ; d=0,33$. Para Evitação, os homens $(M=2,90 ; D P=$ $1,00)$ apresentaram pontuação significativamente superior à das 
Tabela 2

Correlações entre as Dimensões de Apego e Demais Variáveis

\begin{tabular}{|c|c|c|c|c|c|c|c|c|c|c|}
\hline & 1 & 2 & 3 & 4 & 5 & 6 & 7 & 8 & 9 & 10 \\
\hline \multicolumn{11}{|l|}{ 1. Dimensão Ansiedade } \\
\hline 2. Dimensão Evitação & $-0,01$ & & & & & & & & & \\
\hline 3. Idade & $-0,21 * *$ & 0,04 & & & & & & & & \\
\hline 4. Tempo de relacionamento atual & $-0,23 * *$ & $-0,06$ & $0,60 * *$ & & & & & & & \\
\hline $\begin{array}{l}\text { 5. Tempo de relacionamento mais } \\
\text { longo }\end{array}$ & $-0,24 * *$ & $-0,12 * *$ & $0,71^{* *}$ & $0,82 * *$ & & & & & & \\
\hline 6. ETAS: Intimidade & $-0,06$ & $-0,52 * *$ & $-0,11 * *$ & 0,01 & 0,004 & & & & & \\
\hline 7. ETAS: Paixão & $0,14 * *$ & $-0,38 * *$ & $-0,14 * *$ & $-0,07$ & $-0,07$ & $0,59 * *$ & & & & \\
\hline 8. ETAS: Comprometimento & $0,12 * *$ & $-0,50 * *$ & $-0,003$ & $0,12 *$ & $0,10^{*}$ & $0,66^{* *}$ & $0,64 * *$ & & & \\
\hline 9. SOI-R: Comportamento & $-0,06$ & $0,16^{* *}$ & $0,17 * *$ & $-0,15^{* *}$ & $-0,05$ & $-0,16^{* *}$ & $-0,08^{*}$ & $-0,22 * *$ & & \\
\hline 10. SOI-R: Atitude & $-0,16^{* *}$ & $0,15^{* *}$ & 0,01 & $-0,11^{*}$ & $-0,08^{*}$ & $-0,17 * *$ & $-0,11 * *$ & $-0,30 * *$ & $0,55 * *$ & \\
\hline 11. SOI-R: Desejo & $-0,10^{*}$ & $0,26^{* *}$ & $-0,02$ & $-0,14^{* *}$ & $-0,15^{* *}$ & $-0,21 * *$ & $-0,15^{* *}$ & $-0,33^{* *}$ & $0,46^{* *}$ & $0,62 * *$ \\
\hline
\end{tabular}

Nota. ${ }^{*} p<0,05 ; * * p<0,01$. ETA: Escala Triangular do Amor; SOI-R: Inventário de Sociossexualidade Revisado.

mulheres $(M=2,59 ; D P=0,91), F(1,610)=16,0 ; p<0,001$; $d=0,32$.

Por fim, verificou-se a possibilidade do instrumento diferenciar os níveis de apego de participantes engajados em um relacionamento compromissado daqueles envolvidos em um relacionamento sem compromisso, também por meio de uma MANOVA. Observou-se diferença significativa entre os grupos, $\Lambda$ de Wilks $=0,91 ; F(2,449)=22,0 ; p<0,001 ; \eta^{2}=0,09$. Os testes univariados permitiram constatar diferenças tanto para a Ansiedade, $F(1,450)=4,84 ; p=0,028 ; d=0,36$, quanto para a Evitação, $F(1,450)=39,5 ; p<0,001 ; d=1,00$. Em ambas as dimensões, aqueles em um relacionamento sem compromisso (Ansiedade, $M=4,44 ; D P=0,98$; Evitação, $M=3,23 ; D P=$ $0,78)$ diferiram significativamente quando comparados com indivíduos em relacionamento compromissado (Ansiedade, $M=$ 4,04; $D P=1,17$; Evitação, $M=2,43 ; D P=0,83$ ). As diferenças sexuais e entre grupos encontradas estão em consonância com a teoria do construto.

\section{Discussão e conclusão}

Realizou-se a tradução e adaptação para a realidade brasileira do Experience in Close Relationships Inventory (Brennan et al., 1998) e encontraram-se satisfatórias evidências de validade e precisão da versão brasileira do instrumento. Para tanto, conduziram-se dois estudos consecutivos. O primeiro estudo mostrou adequação da maioria dos itens, contudo, também evidenciou a possibilidade de reformulações com vistas ao aperfeiçoamento do instrumento. No Estudo 2, partiu-se dos itens elaborados no primeiro estudo, avaliaram-se suas propriedades psicométricas e voltou-se ao instrumento original a fim de realizar modificações condizentes com o conteúdo de cada item. Realizaram-se alterações em 22 itens e testou-se empiricamente o instrumento reformulado. Após a reformulação, comparada à primeira versão, constataram-se: acréscimo de variância explicada para o instrumento, incrementos nos coeficientes alfas das dimensões e adequação de todos os itens à suas respectivas dimensões de acordo com o instrumento original.

A constante procura por evidências de validade de instrumentos, como defendido pela American Educational Research Association, American Psychological Association e National Council on Measurement in Education (1999), pressupõe uma permanente busca por atualização e aperfeiçoamento dos instrumentos. Essas atualizações e aperfeiçoamentos pautam-se em adaptações à época e a cultura da população sobre a qual a avaliação psicológica será conduzida. Levando-se isso em conta, ressalta-se a importância de levantamentos prévios e adaptações a partir de dados empíricos antes da conclusão de trabalhos de tradução de instrumentos, bem como, na própria elaboração de novos instrumentos. Salienta-se ainda que no caso específico do ECR-Brasil a condução do processo com dois estudos empíricos consecutivos possibilitou consideráveis melhorias ao instrumento e a apresentação de uma versão final mais refinada da escala.

A versão final do ECR-Brasil apresentou duas dimensões que representam a Ansiedade e a Evitação relacionadas ao apego em relacionamentos amorosos/românticos. Cada dimensão é formada por 18 itens, tal como a versão original do instrumento (Brennan et al., 1998). Essa estrutura bifatorial da escala encontrou suporte também em uma análise fatorial confirmatória conduzida em acordo com outros estudos para o ECR revisado (Brenning et al., 2011; Sibley et al., 2005). Assim como na versão original do instrumento e em estudos de tradução conduzidos em outros países, as dimensões apresentaram correlações próximas de zero, sugerindo ortogonalidade do construto (Alonso-Arbiol et al., 2007; Brennan et al., 1998; Mallinckrodt \& Wang, 2004; Paiva \& Figueiredo, 2010; Piccardi et al., 2000). Os índices de consistência interna das dimensões do ECR-Brasil foram satisfatórios e apontam para uma adequada precisão do instrumento (Cronbach, 1951; Nunnally, 1978).

As relações entre as dimensões de apego aferidas pelo ECR-Brasil e as demais variáveis investigadas neste estudo 
mostraram-se em acordo com o esperado pela teoria e em conformidade com estudos anteriores em outras culturas (Collins \& Read, 1990; Hazan \& Shaver, 1987; Madey \& Rodgers, 2009; Schmitt, 2005; Simpson, 1990). Verificou-se que quanto maiores os níveis de Ansiedade e Evitação relacionadas ao apego, que indicam estilo inseguro de apego, menor o tempo de duração dos relacionamentos estabelecidos, seja o atual ou o mais longo da vida, assim como era esperado (Hazan \& Shaver, 1987).

Com relação aos níveis de amor, verificou-se que quanto maiores os escores em Evitação, menores os níveis de intimidade, paixão e comprometimento em relacionamentos amorosos. Esse resultado está em acordo com estudos prévios que encontraram menores níveis de amor e satisfação com o relacionamento, menor comprometimento com o relacionamento e menores níveis de confiança no parceiro entre aqueles do estilo de apego evitativo (Collins \& Read, 1990; Madey \& Rodgers, 2009; Simpson, 1990). Também foram encontradas relações positivas, embora fracas, entre a dimensão Ansiedade do apego e paixão e comprometimento sugerindo que o receio de perder o parceiro romântico atue como um propulsor de níveis de amor apaixonado e de compromisso nos relacionamentos.

No que diz respeito à sociossexualidade, as relações encontradas mostraram que quanto maiores os níveis de Evitação maior a atitude, o desejo e os comportamentos de irrestrição sexual. Pessoas que apresentam orientação sociossexual restrita demonstram maior necessidade de proximidade, envolvimento e comprometimento antes de se engajarem em comportamentos sexuais com alguém, o que reflete uma estratégia reprodutiva de longo prazo (Buss \& Schmitt, 1993; Penke \& Asendorpf, 2008; Simpson \& Gangestad, 1991). Ao contrário, indivíduos apresentando uma orientação sociossexual irrestrita sentem-se confortáveis em fazer sexo sem ter compromisso estabelecido com o parceiro, sendo tal padrão reflexo de estratégias reprodutivas de curto prazo (Buss \& Schmitt, 1993; Penke \& Asendorpf, 2008; Simpson \& Gangestad, 1991).

Assim como Schmitt (2005) encontrou em diversas culturas, os resultados deste estudo apontam para uma associação positiva entre Evitação relacionada ao apego e estratégias de curto prazo em relacionamentos românticos. A utilização de um instrumento de três fatores na medida da sociossexualidade possibilitou a observação, a considerar-se inédita, de correlação ligeiramente mais forte entre o desejo de irrestrição sexual e a dimensão Evitação. Também foram observadas correlações negativas e fracas entre a dimensão Ansiedade e os fatores atitude e desejo da sociossexualidade. Considerando-se uma cultura que preza por relacionamentos monogâmicos e restritos em que os participantes estão inseridos, esses achados sugerem que o receio em perder o parceiro facilita o fortalecimento de crenças normativas de restrição sexual.

Por fim, destaca-se que o instrumento foi capaz de discriminar grupos de pessoas a partir dos níveis de apego. No que diz respeito às diferenças sexuais, as mulheres mostraram média superior em Ansiedade, enquanto os homens em Evitação, tal resultado está em acordo com estudos anteriores (ver metaanálise de Del Giudice, 2011). Esse resultado guarda relações com as diferenças sexuais encontradas em estratégias sexuais (Buss
\& Schmitt, 1993). As mulheres tendem a apresentar uma maior Ansiedade relacionada ao apego quando comparadas aos homens em função de uma contra estratégia adaptativa à maior Evitação masculina, solicitando mais comprometimento e investimento no relacionamento a fim de maximizar o investimento parental (Del Giudice, 2011; Schmitt, 2005). Por outro lado, uma maior Evitação relacionada ao apego entre os homens facilitaria o estabelecimento de múltiplos relacionamentos de curto prazo, aumentando as chances de sucesso reprodutivo(Del Giudice, 2011; Schmitt, 2005). Com relação às diferenças entre aqueles em relacionamento compromissado e não compromissado os resultados estão em consonância com estudos anteriores que sugerem padrões de apego seguro (níveis baixos em Ansiedade e Evitação) associam-se a comprometimento no relacionamento (Collins \& Read, 1990; Hazan \& Shaver, 1987; Madey \& Rodgers, 2009; Simpson, 1990).

A versão brasileira do Experience in Close Relationships Inventory traduzido e adaptado neste estudo mostrou-se em acordo com a versão original do instrumento. Além disso, as evidências encontradas sustentam adequação da escala para aferir o apego adulto a partir de interações em relacionamentos amorosos/românticos. O instrumento afere duas dimensões ortogonais do apego: Ansiedade e Evitação, que combinadas podem caracterizar diferentes estilos de apego. Ressalta-se a importância da realização de novos estudos utilizando o ECRBrasil, sobretudo visando a superar as limitações encontradas neste trabalho, por exemplo, baixa representatividade de algumas regiões do Brasil na amostra.

\section{Referências}

Ainsworth, M. D. S., Blehar, M. C., Waters, E., \& Wall, S. (1978). Patterns of Attachment: A Psychological Study of the Strange Situation. Hillsdale: Erlbaum

Alonso-Arbiol, I., Balluerka, N., \& Shaver, P. (2007). A Spanish version of the Experiences in Close Relationships (ECR) adult attachment questionnaire. Personal Relationships, 14(1), 45-63.

American Educational Research Association, American Psychological Association, \& National Council on Measurement in Education (1999). Standards for educational and psychological testing. Washington: American Educational Research Association Publications.

Bagozzi, R. P., \& Heatherton, T. F. (1994). A general approach to representing multifaceted personality constructs: Application to state self-esteem. Structural Equation Modeling, 1(1), 35-67.

Bartholomew, K., \& Horowitz, L. M. (1991). Attachment styles among young adults: A test of a four-category model. Journal of Personality and Social Psychology, 61(2), 226-244.

Basso, L. A., \& Marin, A. H. (2010). Comportamento de apego em adultos e a experiência da perda de um ente querido. Aletheia, 32, 92-103.

Belsky, J. (1997). Attachment, mating, and parenting: An evolutionary interpretation. Human Nature, 8(4), 361-381.

Bowlby, J. (2002). Apego e perda: Vol I. Apego (3 $3^{\underline{a}}$ ed., Á. Cabral, trad.). São Paulo: Martins Fontes. (Texto original publicado em 1969)

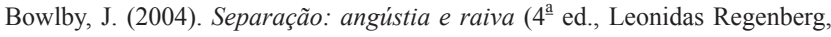
O. S. da Mota \& M. Hegenberg, Trads.). São Paulo: Martins Fontes. (Texto original publicado em 1973)

Brennan, K. A., Clark, C. L., \& Shaver, P. R. (1998). Self-report measurement of adult attachment: An integrative overview. In J. A. Simpson \& W. S. Rholes (Orgs.), Attachment theory and close relationships (pp. 46-76). Nova 
Iorque: Guilford Press.

Brenning, K., Soenens, B., Braet, C., \& Bosmans, G. (2011). An adaptation of the experiences in close relationships scale-revised for use with children and adolescents. Journal of Personal and Social Relationships, 28(8), 1048-1072.

Bretherton, I. (1992). The origins of attachment theory: John Bowlby and Mary Ainsworth. Developmental Psychology, 28(5), 759-775.

Buss, D. M., \& Schmitt, D. P. (1993). Sexual strategies theory: An evolutionary perspective on human mating. Psychological Review, 100(2), 204-232.

Byrne, B. M. (2009). Structural equation modeling with AMOS: Basic concepts, applications, and programming ( $2^{\underline{a}}$ ed.). Nova Iorque: Routledge.

Cassepp-Borges, V., \& Teodoro, M. L. M. (2007). Propriedades psicométricas da versão brasileira da escala triangular do amor de Sternberg. Psicologia: Reflexão e Crítica, 20(3), 513-522.

Chisholm, J. S. (1996). The evolutionary ecology of attachment organization. Human Nature, 7(1), 1-38.

Collins, N. L., \& Read, S. J. (1990). Adult attachment, working models and relationship quality in dating couples. Journal of Personality and Social Psychology, 58(4), 644-663.

Cronbach, L. (1951). Coefficient alpha and the internal structure of tests. Psychometrika, 16(3), 297-334.

Crowell, J. A., Fraley, R. C., \& Shaver, P. R. (2008). Measurement of individual differences in adolescent and adult attachment. In J. Cassidy \& P. R. Shaver (Orgs.), Handbook of Attachment: Theory, Research, and Clinical Applications (pp. 599-634). Nova Iorque: Guilford Press.

Del Giudice, M. (2011). Sex differences in romantic attachment: A meta-analysis. Personality and Social Psychology Bulletin, 37(2), 193-214.

Feeney, J. A. (2008). Adult romantic attachment: Developments in the study of couple relationships. In J. Cassidy \& P. Shaver (Orgs.), Handbook of attachment: Theory, research, and clinical applications (pp. 456-481). Nova Iorque: Guilford Press.

Fonseca, V. R. J. R., Silva, G. A., \& Otta, E. (2010). Relação entre depressão pós-parto e a disponibilidade emocional materna. Cadernos de saúde pública, 26(4), 738-746.

Fraley, R. C., \& Shaver, P. R. (2000). Adult romantic attachment: theoretical developments, emerging controversies, and unanswered questions. Review of General Psychology, 4(2), 132-154.

Griffin, D., \& Bartholomew, K. (1994). Models of the self and other: Fundamental dimensions underlying measures of adult attachment. Journal of Personality and Social Psychology, 67(3), 430-445.

Harlow, H. F. (1958). The Nature of Love. American Psychologist, 13, 673-685. Hazan, C., \& Shaver, P, R. (1987). Romantic love conceptualized as an attachment process. Journal of Personality and Social Psychology, 52(3), 511-524.

Madey, S. F., \& Rodgers, L. (2009). The effect of attachment and Sternberg's triangular theory of love on relationship satisfaction. Individual Differences Research, 7(2), 76-84.

Main, M., \& Solomon, J. (1986). Discovery of a disorganized/disoriented attachment pattern. In T. B. Brazelton \& M. W. Yogman (Orgs.), Affective development in infancy (pp. 95-124). Norwood: Ablex.

Mallinckrodt, B; \& Wang, C-C. (2004). Quantitative methods for verifying semantic equivalence of translated research instruments: A Chinese version of the Experiences in Close Relationships Scale. Journal of Counseling Psychology, 51(3), 368-379.

McDonald, R. P., \& Ho, M. R. (2002). Principles and practice in reporting structural equation analyses. Psychological Methods, 7(1), 64-82.

Nunnally, J. C. (1978). Psychometric theory ( $2^{\underline{a}}$ ed.). Nova Iorque: McGraw Hill.

Paiva, C. A., \& Figueiredo, B. (2010). Study of validation of the Portuguese version of the inventory Experiences in Close Relationships. Interpersona, $4(2), 237-270$.

Pasquali, L. (2005). Análise fatorial para pesquisadores. Brasília: LabPAM.

Penke, L., \& Asendorpf, J. B. (2008). Beyond global sociosexual orientations: A more differentiated look at sociosexuality and its effects on courtship and romantic relationships. Journal of Personality and Social Psychology, 95(5), 1113-1135.

Picardi, A., Bitetti, D., Puddu, P., \& Pasquini, P. (2000). La scala "Experiences in close relationships" (ECL), un nuovo strumento per la valutazione dell'attaccamento negli adulti: traduzione, adattamento e validazione della versione italiana. Rivista di Psichiatria, 35(3), 114-120.

Schmitt, D. P. (2005). Is short-term mating the maladaptive result of insecure attachment? A test of competing evolutionary perspectives. Personality and Social Psychology Bulletin, 31(6), 747-768.

Sibley, C. G., Fischer, R., \& Liu, J. H. (2005). Reliability and validity of the Revised Experiences in Close Relationships (ECR-R) self-report measure of adult romantic attachment. Personality and Social Psychology Bulletin, 31(11), 1524-1536.

Simpson, J. A. (1990). Influence of attachment styles on romantic relationships. Journal of Personality and Social Psychology, 59(5), 971-980.

Simpson, J. A., \& Gangestad, S. W. (1991). Individual differences in sociosexuality: Evidence for convergent and discriminant validity. Journal of Personality and Social Psychology, 60(6), 870-883.

Sternberg, R. J. (1997). Construct validation of a triangular love scale. European Journal of Psychology, 27(3), 313-335.

Victor Kenji Medeiros Shiramizu, doutorando do Programa de Pós-graduação em Psicobiologia da Universidade Federal do Rio Grande do Norte, é biólogo formado pela Universidade Federal do Rio Grande do Norte. Endereço para correspondência: Universidade Federal do Rio Grande do Norte, Centro de Biociências, Campus Universitário, Bairro Lagoa Nova - Natal, RN - Brasil - CEP 59078-970 - Caixa-postal: 1511. Telefone: (84) 3215-3409, Ramal: 213. Fax: (84) 32119206. E-mail: victorshiramizu@gmail.com Jean Carlos Natividade, doutorando do Programa de Pós-Graduação em Psicologia da Universidade Federal do Rio Grande do Sul, é mestre em Psicologia pela Universidade Federal de Santa Catarina. E-mail: jeannatividade@gmail.com

Fívia de Araújo Lopes, doutora em Psicobiologia pela Universidade Federal do Rio Grande do Norte, é professora Associada II no Departamento de Fisiologia e docente do Programa de Pós-graduação em Psicobiologia da Universidade Federal do Rio Grande do Norte. E-mail: fivialopes@gmail.com 